\title{
Multi-User Preemptive Scheduling For Critical Low Latency Communications in 5G Networks
}

\author{
Ali A. Esswie ${ }^{1,2}$, Member, IEEE, and Klaus I. Pedersen ${ }^{1,2}$, Senior Member, IEEE \\ ${ }^{1}$ Nokia Bell-Labs, Aalborg, Denmark \\ ${ }^{2}$ Department of Electronic Systems, Aalborg University, Denmark \\ Email: ali.esswie@nokia.com
}

\begin{abstract}
G new radio is envisioned to support three major service classes: enhanced mobile broadband (eMBB), ultra-reliable low-latency communications (URLLC), and massive machine type communications. Emerging URLLC services require up to one millisecond of communication latency with $99.999 \%$ success probability. Though, there is a fundamental trade-off between system spectral efficiency (SE) and achievable latency. This calls for novel scheduling protocols which cross-optimize system performance on usercentric; instead of network-centric basis. In this paper, we develop a joint multi-user preemptive scheduling strategy to simultaneously cross-optimize system SE and URLLC latency. At each scheduling opportunity, available URLLC traffic is always given higher priority. When sporadic URLLC traffic appears during a transmission time interval (TTI), proposed scheduler seeks for fitting the URLLC-eMBB traffic in a multiuser transmission. If the available spatial degrees of freedom are limited within a TTI, the URLLC traffic instantly overwrites part of the ongoing eMBB transmissions to satisfy the URLLC latency requirements, at the expense of minimal eMBB throughput loss. Extensive dynamic system level simulations show that proposed scheduler provides significant performance gain in terms of eMBB SE and URLLC latency.

Index Terms - URLLC; 5G; MU-MIMO; Channel hardening; RRM; Preemptive scheduling.
\end{abstract}

\section{INTRODUCTION}

The standardization of the fifth generation $(5 \mathrm{G})$ new radio (NR) is progressing with big momentum within the 3rd generation partnership project (3GPP) community, to release the first $5 \mathrm{G}$ specifications [1-3]. Ultra-reliable and low-latency communications (URLLC) is envisioned as a key requirement of the 5G-type communications, to support broad categories of many new applications from wireless industrial control, autonomous driving, and to tactile internet [4]. URLLC services require stringent latency and reliability levels, e.g., $1 \mathrm{~ms}$ at the $1-10^{-5}$ reliability level [5]. Such a challenging latency limit denotes that a URLLC packet which can not be transmitted and successfully decoded before the URLLC latency deadline, is considered as information-less and of no-use.

Simultaneously achieving the requirements of extreme spectral efficiency (SE) for enhanced mobile broadband (eMBB) services and ultra-low latency for URLLC applications is a challenging problem [6]. Achieving such
URLLC latency demands more radio resources with ultralow target block error rate (BLER); though, it leads to a significant loss in the network SE. Also, reserving dedicated resources for URLLC traffic is spectrally inefficient due to its sporadic nature.

To meet the stringent URLLC requirements, various studies have been recently presented in the open literature. User-specific scheduling with flexible transmission time intervals (TTIs) $[7,8]$ is recognized as an enabler to achieve the URLLC latency limit, e.g., URLLC traffic with a short TTI and eMBB with a longer TTI. However, the former increases the aggregate overhead of the control channel. Additionally, different configurations of microscopic and macroscopic diversity [9] are proven beneficial for URLLC to significantly reduce the outage probability of the signal-to-interference-noise-ratio (SINR). Advanced medium access control enhancements [10] are also reported towards optimized scheduling of URLLC traffic, including link adaptation filtering in partly-loaded cells, dynamic and load-dependent BLER optimization. Furthermore, preemptive scheduling $[11,12]$ is recently studied to instantly schedule URLLC traffic within a shared channel, monopolized by an ongoing eMBB transmission. Compared to existing studies, achieving the URLLC latency requirements comes at the expense of a degraded SE, e.g., high degrees of macroscopic diversity. Needless to say that a flexible and multi-objective scheduling algorithm, which captures the maximal system degrees of freedom (DoFs), is critical to reach the best achievable URLLCeMBB multiplexing gain.

In this paper, a multi-user preemptive scheduling (MUPS) strategy for densely populated $5 \mathrm{G}$ networks is proposed. MUPS aims to simultaneously cross-optimize the network SE and URLLC latency. At each scheduling TTI, MUPS scheduler assigns URLLC traffic a higher priority for immediate scheduling without buffering. If sporadic URLLC traffic arrives at the $5 \mathrm{G}$ general NodeB (gNB) during an arbitrary TTI, the gNB first attempts to fit the URLLC packets within an ongoing eMBB transmission. If the spatial DoFs are insufficient, the gNB decides to immediately overwrite, i.e., preemptively schedule (PS), the physical resource blocks (PRBs) over which URLLC users reported the best received SINR. Compared to conventional PS scheduler, proposed MUPS utilizes the spatial DoFs, offered by the transmit antenna array, to 
extract the best achievable multiplexing gain, satisfying both: URLLC latency budget and eMBB throughput requirements.

Due to the complexity of the $5 \mathrm{G}$ NR system and the addressed problems, performance evaluation is validated using advanced system level simulations which offer high degree of realism and ensure reliable statistical results. Those simulations are based on widely accepted models and being calibrated with the $3 \mathrm{GPP} 5 \mathrm{G}$ NR assumptions [1-3].

This paper is organized as follows. Section II presents the system model. Section III outlines the problem formulation and proposed MUPS scheduler. Performance analysis appears in Section IV and the paper is concluded in Section V.

\section{System MODEL}

We consider a downlink (DL) multi-user multiple-input multiple-output (MU-MIMO) system, with $C$ cells. Each cell is equipped with $N_{t}$ transmit antennas while there are $K$-uniformly-distributed users per cell, each with $M_{r}$ receive antennas. Users are dynamically multiplexed through orthogonal frequency division multiple access (OFDMA), and with $15 \mathrm{KHz}$ sub-carrier spacing. There are two types of DL traffic under evaluation: (1) URLLC time-sporadic traffic of $Z$-bit finite payload per user with a Poisson point arrival process $\lambda$, and (2) eMBB full buffer traffic with infinite payload. The cell loading condition is described by $K_{U R L L C}+K_{e M B B}=K$, where $K_{U R L L C}$ and $K_{e M B B}$ denote the average number of URLLC and eMBB users per cell, respectively. URLLC traffic is scheduled with a short TTI of 2 OFDM symbols (mini-slot of $0.143 \mathrm{~ms}$ ) to meet the URLLC latency budget [1]. However, eMBB users are scheduled with a long TTI of 14 OFDM symbols (slot of $1 \mathrm{~ms})$ to maximize system $\mathrm{SE}$.

A maximum MU subset $G \in K$, where $G_{c} \leq N_{t}$ is allowed per PRB per cell, with equal power sharing. Thus, the received DL signal at the $k^{t h}$ user from the $c^{t h}$ cell is given by

$$
\begin{aligned}
\mathrm{y}_{k, c} & =\mathbf{H}_{k, c} \mathbf{V}_{k, c} s_{k, c}+\sum_{g \in G_{c}, g \neq k} \mathbf{H}_{k, c} \mathbf{V}_{g, c} s_{g, c} \\
& +\sum_{j=1, j \neq c}^{C} \sum_{g \in G_{j}} \mathbf{H}_{g, j} \mathbf{V}_{g, j} s_{g, j}+\mathbf{n}_{k},
\end{aligned}
$$

where $\mathbf{H}_{k, c} \in \mathcal{C}^{M_{r} \times N_{t}}, \forall k \in\{1, \ldots, K\}, \forall c \in\{1, \ldots, C\}$ is the 3GPP spatial channel matrix seen by the $k^{\text {th }}$ user from the $c^{\text {th }}$ cell, $\mathbf{V}_{k, c} \in \mathcal{C}^{N_{t} \times 1}$ and $s_{k, c}$ are the precoding vector (assuming a single stream transmission) and the transmitted symbol, respectively. $\mathbf{n}_{k}$ is the additive Gaussian white noise at the $k^{\text {th }}$ user. The first summation in eq. (1) stands for the inter-user interference and the second considers the inter-cell interference. The received signal after applying the antenna combining vector $\mathbf{U}_{k, c} \in \mathcal{C}^{M_{r} \times 1}$ is given by

$$
\mathrm{y}_{k, c}^{*}=\left(\mathbf{U}_{k, c}\right)^{\mathrm{H}} \mathrm{y}_{k, c},
$$

where $(.)^{\mathrm{H}}$ indicates the Hermitian transpose. The antenna combining vector is designed based on the linear minimum mean square error interference rejection combining (LMMSE-IRC) criteria [13], in order to project the received signal on a signal subspace which minimizes the MSE, given by

$$
\mathbf{U}_{k, c}=\left(\mathbf{H}_{k, c} \mathbf{V}_{k, c}\left(\mathbf{H}_{k, c} \mathbf{V}_{k, c}\right)^{\mathrm{H}}+\mathrm{W}\right)^{-1} \mathbf{H}_{k, c} \mathbf{V}_{k, c}
$$

where $\mathrm{W}=\mathbb{E}\left(\mathbf{H}_{k, c} \mathbf{V}_{k, c}\left(\mathbf{H}_{k, c} \mathbf{V}_{k, c}\right)^{\mathrm{H}}\right)+\sigma^{2} \mathrm{I}_{M_{r}}$ is the interference covariance matrix, $\mathbb{E}($.$) denotes the statistical$ expectation, and $\mathrm{I}_{M_{r}}$ is $M_{r} \times M_{r}$ identity matrix. The received SINR at the $k^{t h}$ user can be expressed as

$\Upsilon_{k, c}=\frac{p_{k}^{c}\left|\mathbf{H}_{k, c} \mathbf{V}_{k, c}\right|^{2}}{1+\sum_{g \in G_{c}, g \neq k} p_{g}^{c}\left|\mathbf{H}_{k, c} \mathbf{V}_{g, c}\right|^{2}+\sum_{j \in C, j \neq c} \sum_{g \in G_{j}} p_{g}^{j}\left|\mathbf{H}_{g, j} \mathbf{V}_{g, j}\right|^{2}}$,

where $p_{k}^{c}$ is the transmission power of the $k^{\text {th }}$ user in the $c^{t h}$ cell. The per-user per-PRB data rate can then be calculated as,

$$
r_{k, r b}=\log _{2}\left(1+\frac{1}{\eta_{c}} \Upsilon_{k, c}\right)
$$

where $\eta_{c}=\operatorname{card}\left(G_{c}\right)$ is the MU rank on this PRB.

Moreover, the link adaptation of the data transmission is based on the frequency-selective channel quality indication (CQI) reports to satisfy a target BLER. However, the CQI reports from the MU pairs can be misleading since the calculation of the inter-user interference and power sharing are not considered in the CQI estimation. Hence, to stabilize the link adaptation process against MU variance, an offset of $\delta \mathrm{dB}$ is applied to the single-user (SU) CQI values before the modulation and coding scheme (MCS) level is selected,

$$
\Gamma_{\mathrm{MU}}=\Gamma_{\mathrm{SU}}-\delta
$$

where $\Gamma_{\mathrm{MU}}$ and $\Gamma_{\mathrm{SU}}$ are the updated $\mathrm{MU}$ and reported CQI levels, respectively. Additionally, due to the bursty nature of the URLLC traffic, it sporadically destabilizes the reported CQI levels [10], especially when an MU transmission is not possible due to the fast varying interference patterns; otherwise, the interference from the co-scheduled users contributes to stabilizing the URLLC CQI levels. Thus, we further apply a sliding filter, e.g., a low pass filter, in order to smooth the instantaneous variation rate of the CQI levels as follows,

$$
\partial(t)=\xi \Gamma_{\mathrm{MU}}+(1-\xi) \partial(t-1),
$$

where $\partial(t)$ is the MU CQI value to be considered for link adaptation and MCS selection at the $t^{t h}$ TTI, and $\xi \leq 1$ is a tunable coefficient to specify how much weight should be given to current reported CQI value. 


\section{Proposed Multi-User Preemptive Scheduling}

In this section, the concept of the proposed MUPS scheduler is introduced. Under the $5 \mathrm{G}$ umbrella, there are multi user-specific, instead of network-specific, objectives which need to be fulfilled simultaneously, e.g., eMBB SE maximization, URLLC latency and BLER minimization as follows,

$$
\begin{gathered}
\forall k_{e M B B} \in \mathcal{K}_{e M B B}: \arg \max _{\mathcal{K}_{e M B B}} \sum_{k_{e M B B}}^{K_{e M B}} \sum_{r b \in R B_{k}} r_{k, r b}, \\
\forall k_{U R L L C} \in \mathcal{K}_{U R L L C}: \arg \min _{\mathcal{K}_{U R L L C}}(\beta), \beta \leq 1 \mathrm{~ms}, \\
\forall k \in \mathcal{K}: \arg \min _{\mathcal{K}}(\psi),
\end{gathered}
$$

where $\mathcal{K}_{e M B B}$ and $\mathcal{K}_{U R L L C}$ denote the set of active eMBB and URLLC users, respectively. $\beta$ and $\psi$ indicate the URLLC latency at the $1-10^{-5}$ reliability level and user BLER, respectively. This is a challenging and non-trivial optimization problem, e.g., achieving Shannon SE requires infinite latency budget. The proposed MUPS aims at achieving the maximum possible system SE, while at the same time preserving the URLLC required latency.

As shown in Fig. 1, if there is no incoming URLLC traffic at an arbitrary TTI, MUPS assigns SU dedicated resources to incoming or buffered eMBB traffic based on the proportional fair $(\mathrm{PF})$ criteria as

$$
\begin{gathered}
\Theta_{\mathrm{PF}}=\frac{r_{k, r b}}{\overline{r_{k, r b}}}, \\
k_{e M B B}^{*}=\arg \max _{\mathcal{K}_{e M B} \Theta_{\mathrm{PF}}},
\end{gathered}
$$

where $\overline{r_{k, r b}}$ is the average delivered data rate of the $k^{t h}$ user. If incoming URLLC traffic is aligned at the start of the current TTI, e.g., either it is a short URLLC or long eMBB TTI, MUPS applies the weighted PF (WPF) criteria to instantly schedule URLLC traffic with a higher priority on available resources as given by

$$
\Theta_{\mathrm{WPF}}=\frac{r_{k, r b}}{\overline{r_{k, r b}}} \alpha
$$

where $\alpha$ is the scheduling coefficient and $\alpha_{U R L L C} \gg$ $\alpha_{e M B B}$. Afterwards, MUPS schedules pending or new eMBB traffic on remaining resources.

If URLLC traffic arrives at the gNB during an eMBB TTI transmission while scheduling resources are not available, gNB attempts to dynamically multiplex the incoming short-TTI URLLC users within the ongoing long-TTI eMBB transmissions, if there are sufficient spatial DoFs on this TTI. The spatial DoFs represent the ability to jointly process several signals between different sets of transmitters and receivers, if corresponding channels are highly uncorrelated. Accordingly, URLLC users experience no buffering overhead and then the URLLC latency budget can be satisfied. If a successful pairing, i.e., MU URLLCeMBB transmission over an arbitrary PRB, is not possible,

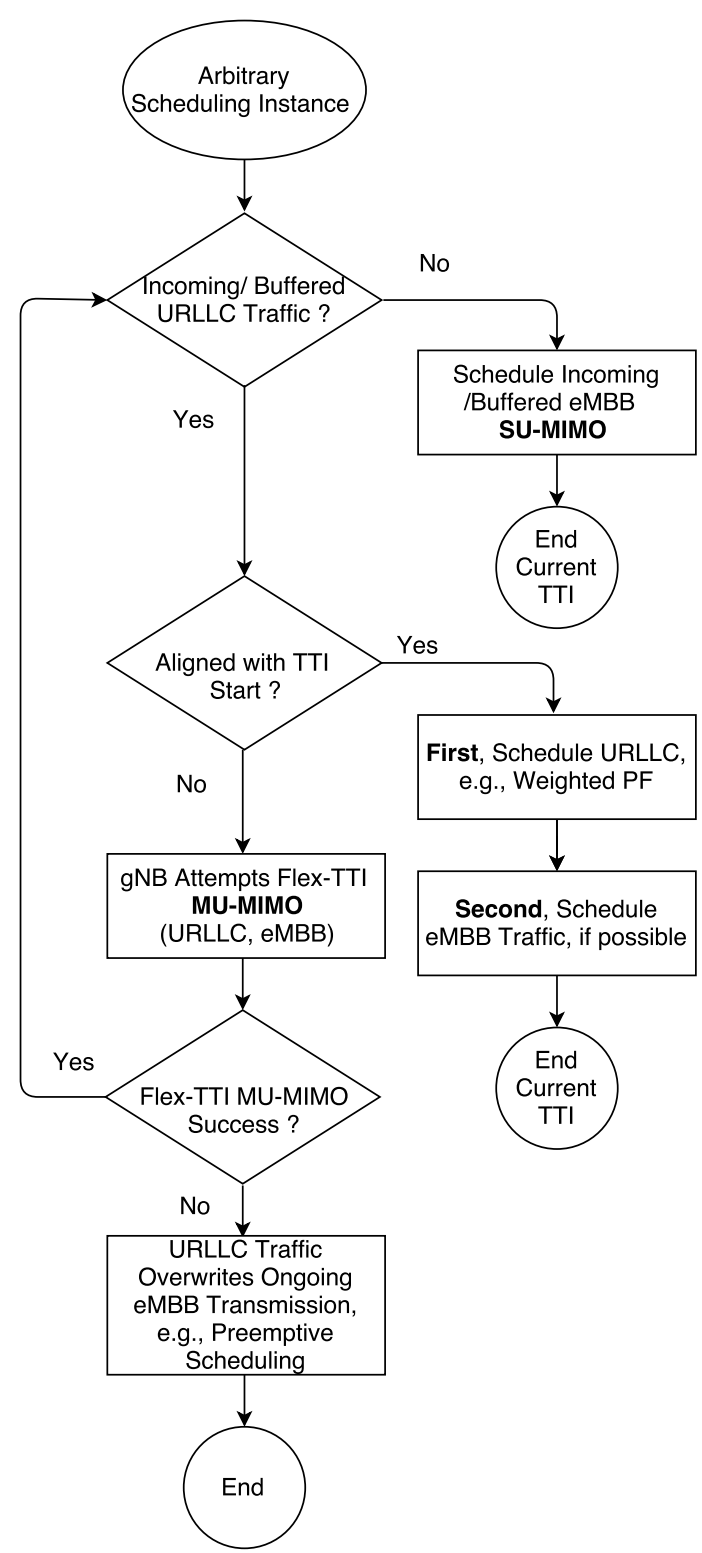

Fig. 1. Flow diagram of proposed MUPS scheduler.

gNB will instantly overwrite the best reported PRBs, known from the URLLC CQI reports, with the incoming URLLC traffic. Thus, victim eMBB transmissions will exhibit a throughput loss.

For $N_{t}=8$ transmit antennas at the gNB, dual codebooks are defined in LTE-Pro standards [14] for DL channel quantization at the user's side, and are given by

$$
\begin{aligned}
& \boldsymbol{\Lambda}_{1}=\left\{\boldsymbol{v}_{1,1}, \boldsymbol{v}_{1,2} \ldots, \boldsymbol{v}_{1,2^{B_{1}}}\right\}, \\
& \boldsymbol{\Lambda}_{2}=\left\{\boldsymbol{v}_{2,1}, \boldsymbol{v}_{2,2} \ldots, \boldsymbol{v}_{2,2^{B_{2}}}\right\},
\end{aligned}
$$

where $\boldsymbol{v}_{i, j}$ denotes the $j^{\text {th }}$ codeword of the $i^{\text {th }}$ codebook, $B_{1}$ and $B_{2}$ are the numbers of bits of the two precoding matrix indices, reported from each user for the gNB to select one codeword from each codebook. Each user projects its estimated DL channel on both codebooks to select the closest possible codewords as 


$$
\begin{aligned}
& \widehat{\boldsymbol{v}}_{1}=\arg \max _{\boldsymbol{v}_{1} \in \boldsymbol{\Lambda}_{1}}\left\|\hat{\mathbf{H}} \boldsymbol{\Lambda}_{1}\right\|^{2}, \\
& \widehat{\boldsymbol{v}}_{2}=\arg \max _{\boldsymbol{v}_{2} \in \boldsymbol{\Lambda}_{2}}\left\|\hat{\mathbf{H}} \boldsymbol{\Lambda}_{2}\right\|^{2},
\end{aligned}
$$

where $\|$.$\| denotes the 2-norm operation. The final pre-$ coding vector at the gNB is obtained by the spatial multiplication of both precoders, and is given by

$$
\mathbf{V}=\widehat{\boldsymbol{v}}_{1} \times \widehat{\boldsymbol{v}}_{2} .
$$

For a MU transmission on a given PRB, the zeroforcing ( $\mathrm{ZF}$ ) beamforming is used to null the inter-user interference between the co-scheduled pairs as expressed by

$$
\begin{gathered}
\mathbf{V}_{\mathrm{MU}}=\left[\mathbf{V}_{1} \ldots \mathbf{V}_{\mathrm{G}}\right], \\
\mathbf{V}_{\mathrm{zf}}=\mathbf{V}_{\mathrm{MU}}\left(\mathbf{V}_{\mathrm{MU}}^{\mathrm{H}} \mathbf{V}_{\mathrm{MU}}\right)^{-1} \operatorname{diag}(\sqrt{P}),
\end{gathered}
$$

where $\mathbf{V}_{\mathrm{G}}$ and $\mathbf{V}_{\mathrm{zf}}$ present the precoder of the $g^{\text {th }}$ user enrolled in a MU-MIMO transmission and the ZF beamforming matrix, where its column vectors are the data beamforming vectors of the MU pairs. The MU transmission success is based on the maximization of the Chordal distance between the ZF beamformers of the co-scheduled users as follows,

$$
\arg \max _{\mathrm{V}_{\mathrm{eMBB}} \in \mathcal{V}_{\mathrm{eMBB}}} \mathbf{d}\left(\mathrm{V}_{\mathrm{URLLC}}, \mathrm{V}_{\mathrm{eMBB}}\right),
$$

where $\mathcal{V}_{\text {eMBB }}$ represents the set of ZF precoders of the eMBB active user set. The Chordal distance is calculated as

$\mathbf{d}\left(\mathrm{V}_{\text {URLLC }}, \mathrm{V}_{\mathrm{eMBB}}\right)=\frac{1}{\sqrt{2}}\left\|\mathrm{~V}_{\text {URLLC }} \mathrm{V}_{\text {URLLC }}^{\mathrm{H}}-\mathrm{V}_{\mathrm{eMBB}} \mathrm{V}_{\mathrm{eMBB}}^{\mathrm{H}}\right\|$.

Upon MU pairing success, the aggregate achievable data rate on a given PRB $r_{r b}$ is expressed by the sum rate of both co-scheduled URLLC and eMBB users as

$$
r_{r b}=\left(r_{e M B B}+r_{U R L L C}-\Delta\right),
$$

where $\Delta$ represents the eMBB and URLLC SU rate loss due to the MU inter-user interference. If a MU pairing is not possible, due to either insufficient spatial DoFs or low number of active eMBB users, the URLLC traffic immediately overwrites the PRBs over which it experiences the best CQI levels. Thus, the eMBB users which have ongoing transmissions on these PRBs suffer from throughput degradation. However, recovery mechanisms can be arbitrarily considered not to include these PRBs as part of the HARQ chase combining process and propagate errors, e.g., consider these PRBs as information-less. Then,

\begin{tabular}{|c|c|}
\hline Parameter & Value \\
\hline Environment & $\begin{array}{l}3 \mathrm{GPP}-\mathrm{UMA}, 7 \mathrm{gNBs}, 21 \text { cells, } \\
500 \text { meters inter-site distance }\end{array}$ \\
\hline Channel bandwidth & $10 \mathrm{MHz}$, FDD \\
\hline gNB antennas & 8,16 and $64 \mathrm{Tx}, 0.5 \lambda$ \\
\hline User antennas & $2,8,16$ and $64 \mathrm{Rx}, 0.5 \lambda$ \\
\hline User dropping & $\begin{array}{l}\text { uniformly distributed } \\
\text { URLLC: } 5 \text { and } 10 \text { users/cell } \\
\text { eMBB: } 5,10 \text { and } 20 \text { users/cell }\end{array}$ \\
\hline User receiver & LMMSE-IRC \\
\hline TTI configuration & $\begin{array}{l}\text { URLLC: } 0.143 \mathrm{~ms} \text { (2 OFDM symbols) } \\
\text { eMBB: } 1 \mathrm{~ms} \text { (14 OFDM symbols) }\end{array}$ \\
\hline MAC scheduler(s) & $\begin{array}{l}\text { URLLC: WPF, SU/MU-MIMO and PS } \\
\text { eMBB: PF, and SU/MU-MIMO }\end{array}$ \\
\hline CQI & periodicity: $5 \mathrm{~ms}$, with $2 \mathrm{~ms}$ latency, $\xi=0.01$ \\
\hline HARQ & $\begin{array}{l}\text { asynchronous HARQ, chase combining } \\
\text { HARQ round trip time }=4 \text { TTIs }\end{array}$ \\
\hline Link adaptation & $\begin{array}{c}\text { dynamic MCS } \\
\text { target URLLC BLER : } 1 \% \\
\text { target eMBB BLER : } 10 \%\end{array}$ \\
\hline Traffic model & $\begin{array}{l}\text { URLLC: bursty, } Z=50 \text { bytes, } \lambda=250 \\
\text { eMBB: full buffer }\end{array}$ \\
\hline MU-MIMO setup & $\begin{array}{l}\text { MU beamforming : ZF } \\
\text { MU rank }(\eta): 2 \\
\text { CQI offset }(\delta): 3 \mathrm{~dB}\end{array}$ \\
\hline Link to system mapping & Mean mutual information per coded bit [11] \\
\hline
\end{tabular}
the sum rate on victim PRBs can be expressed only by the achievable URLLC rate as

$$
r_{r b}=r_{U R L L C} .
$$

Table I

Simulation Parameters.

For the sake of a fair URLLC latency evaluation, we compare the MUPS performance with the preemptiveonly scheduling (PS) [11], where incoming URLLC traffic always overwrites ongoing eMBB transmissions without buffering, at the expense of the system SE. As it will be discussed in Section IV, we demonstrate that a conservative multi-TTI MU-MIMO transmission can be an attractive solution to approach both URLLC latency and eMBB SE requirements.

\section{Simulation Results}

Extensive dynamic system level simulations have been conducted, following the $5 \mathrm{G}$ NR specifications in 3GPP [3]. The major simulation parameters are listed in Table 1, where the baseline antenna setup is $8 \times 2$ unless otherwise mentioned.

Fig. 2 shows the empirical complementary cumulative distribution function (CCDF) of the URLLC latency statistics. We define the cell loading state by $\Omega=$ $\left(K_{e M B B}, K_{U R L L C}\right)$, where the aggregate URLLC offered load per cell in bits/s is calculated as: $K_{U R L L C} \times \lambda \times Z$. Looking at the URLLC latency at the $10^{-5}$ level, both proposed MUPS and PS schedulers achieve the 1-ms limit with $\Omega=(5,5)$. By increasing the system loading, e.g., $K_{e M B B}=10$ and $K_{U R L L C}=10$, the inter-cell interference becomes a dominant component and hence, all schedulers suffer from throughput and latency degradation. Though, MUPS scheduler still shows a decent URLLC latency performance, e.g., $1.7 \mathrm{~ms}$ at $10^{-5}$ level.

PF scheduler suffers from URLLC latency error floor since both URLLC and eMBB users have the same scheduling priority, thus, URLLC large queuing delays occur. WPF shows optimized URLLC latency; however, it 


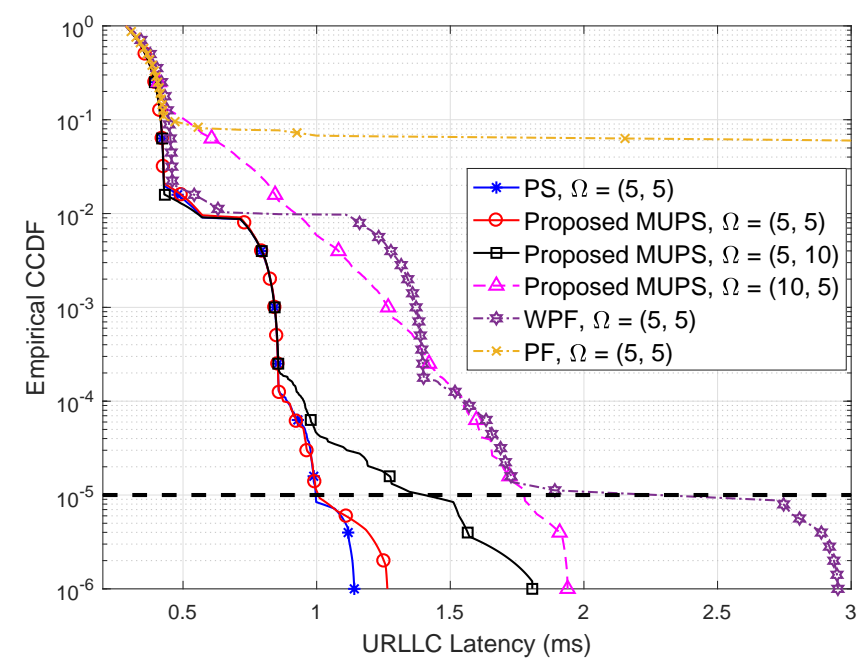

Fig. 2. URLLC latency of MUPS, PS, PF and WPF schedulers.

doesn't achieve the 1-ms limit since the sporadic URLLC traffic, which is available during an eMBB TTI transmission, is buffered, i.e., not scheduled instantly, until the next available TTI opportunity.

Fig. 3 shows the empirical CDF of the average cell throughput in Mbps of the proposed MUPS and PS schedulers under different loading conditions. Under all cell loading states, the MUPS scheduler shows significant gain over PS scheduler, e.g., 26.54\% gain with $\Omega=(20,5)$. MUPS scheduler exhibits a better system SE due to: (1) the successful multi-TTI MU transmissions, and (2) reduction in the number of the experienced PS scheduling events. For the same number of the URLLC users $K_{U R L L C}$, increasing the number of eMBB users $K_{e M B B}$ significantly enhances the MU DoFs, hence, an incoming URLLC user has higher probability to experience an immediate MU pairing success, without falling back to SE-less-efficient PS scheduling. Under such high $K_{e M B B}$ loading, MUPS scheduler attempts many MU pairing success events; however, with limited MU gain due to the aggregate level of inter-cell interference and the higher buffering time. Thus, we also consider a modified version of the MUPS scheduler, denoted as conservative MUPS (C-MUPS), where the URLLC-eMBB pairing success becomes more restricted by the user spatial separation as

$$
\left|\angle\left(\mathrm{V}_{\mathrm{URLLC}}\right)-\angle\left(\mathrm{V}_{\mathrm{eMBB}}\right)\right|^{o} \geq \theta
$$

where $\theta$ is a predefined spatial separation threshold. Thus, C-MUPS achieves lower number of MU attempts with further significant $\mathrm{MU}$ gain, e.g., $~ 62 \%$ gain in average cell throughput with $\Omega=(20,5)$ and $\theta=60^{\circ}$, as shown in Fig. 3.

As depicted in Fig. 4, it shows the average achievable MU throughput increase with respect to average SU throughput. As can be noticed, increasing $K_{U R L L C}$ offers limited DoFs due to the short TTI length of the URLLC users. Furthermore, increasing the URLLC load results in

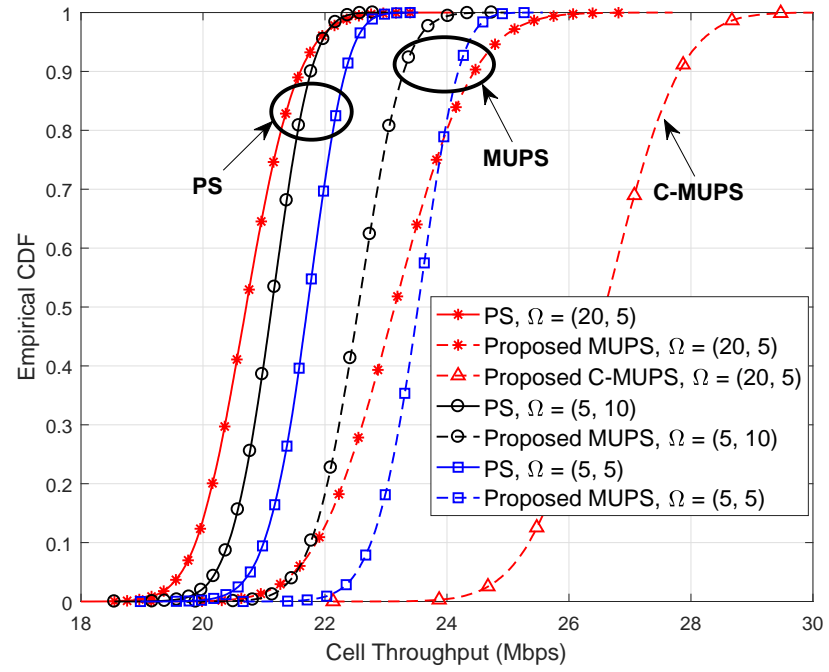

Fig. 3. Cell throughput of MUPS, C-MUPS and PS schedulers.

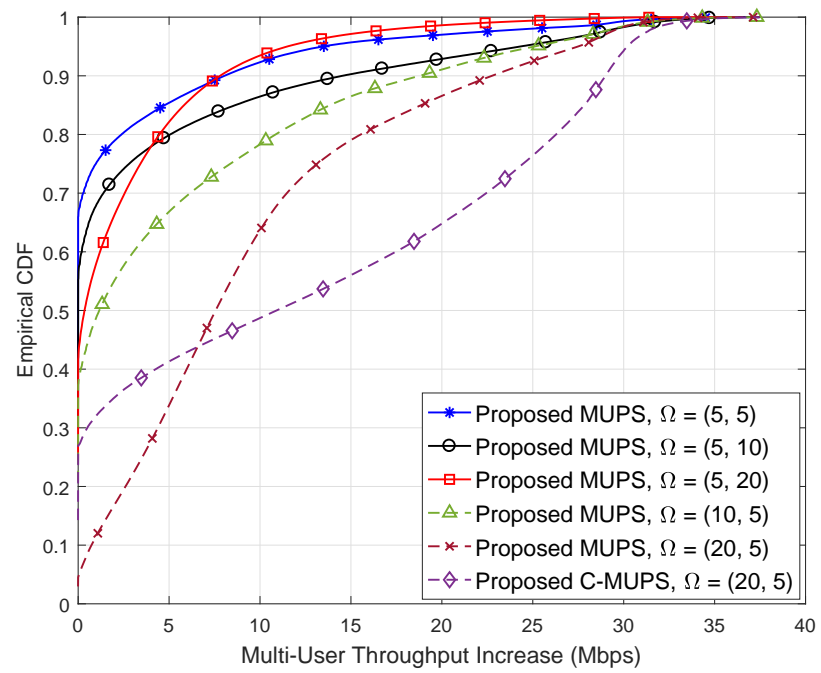

Fig. 4. MU throughput of the MUPS and C-MUPS schedulers.

more sporadic packet arrivals and hence, destabilizing the link adaptation. Increasing the eMBB load offers great spatial DoFs per each URLLC user. With C-MUPS, it shows that less MU success events are experienced, e.g., $72 \%$ instead of $95 \%$ for MUPS with $\Omega=(20,5)$; however, further higher MU throughput is achieved.

Examining the eMBB user performance, Fig. 5 presents a comparison of the eMBB average user throughput. Proposed scheduler shows improved eMBB user throughput, under all loading conditions. The gain in the eMBB user throughput is strongly dependent on the levels of inter-cell and inter-user interference. With light loading conditions, e.g., $\Omega=(5,5)$, the MUPS scheduler experiences few successful pairings with sub-optimal MU gain because of the insufficient available spatial DoFs, e.g., due to the low value of $K_{e M B B}$. On the opposite, under heavy loading conditions, e.g., $\Omega=(20,5)$, MUPS achieves a higher 


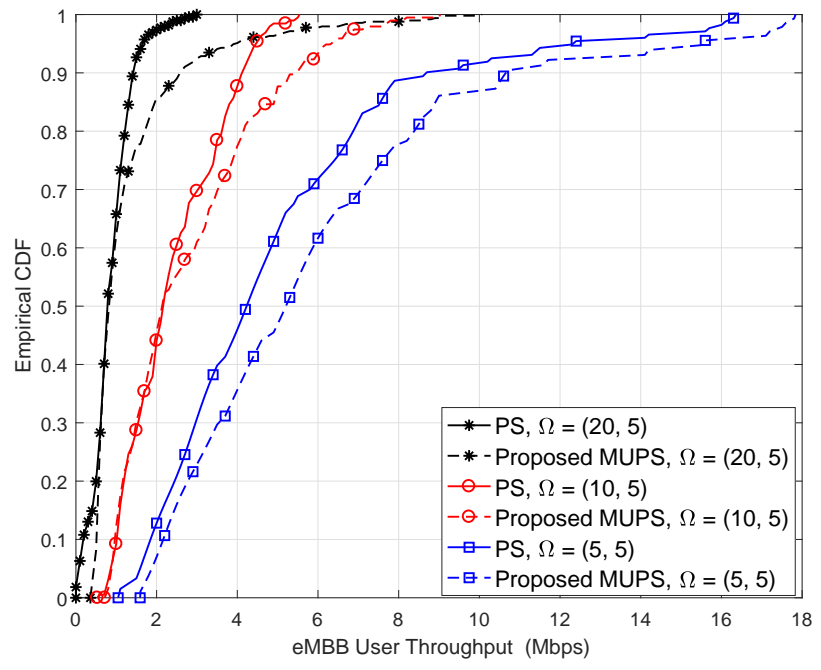

Fig. 5. eMBB user throughput of the MUPS and PS schedulers.

number of successful MU pairings with higher MU gain as the qr experiences few successful pairings with sub-optimal MU gain because of the insufficient available spatial DoFs, e.g., due to the low value of $K_{e M B B}$. On the opposite, under heavy loading conditions, e.g., $\Omega=(20,5)$, MUPS achieves a higher number of successful MU pairings with higher MU gain as the quality of the MU transmission enhances with the number of active eMBB users $K_{e M B B}$.

Interestingly, the MU performance can be further improved with a larger number of antennas, equipped at both transmitter and receiver. Channel hardening $[15,16]$ denotes a fundamental channel phenomenon where the variance of the channel mutual information shrinks as the number of antennas grows,

$$
\sigma^{2}=\frac{1}{\min \left(N_{t}, M_{r}\right)}\left(\frac{\|\mathbf{H}\|^{2}}{\mathbb{E}\left(\|\mathbf{H}\|^{2}\right)}\right) .
$$

Consequently, the fading channel starts to act as a non-fading channel where the channel eigenvalues become less sensitive to the actual distribution of the channel entries. Thus, the channel hardens and becomes much more directional on desired paths with less leakage on the interfering paths, as shown in Fig. 6. As a result, both MU and URLLC performance can be significantly improved.

Fig. 7 introduces the received user SINR in dB, sampled over both URLLC and eMBB users with $\Omega=(20,5)$. For a fair performance comparison, each user is assumed to feedback its serving cell with the exact channel entries without quantization, since there is no a standard quantization codebook for $N_{t}>8$ and $M_{r}>8$. The channel is decomposed and fed-back by the singular value decomposition [17] as: $\mathbf{H}=\boldsymbol{U} \boldsymbol{\Sigma} \mathcal{V}^{\mathrm{H}}$, where $\mathcal{U} \in \mathcal{C}^{M_{r} \times M_{r}}$ and $\mathcal{V} \in \mathcal{C}^{N_{t} \times N_{t}}$ are unitary matrices and $\boldsymbol{\Sigma} \in \mathfrak{N}^{M_{r} \times N_{t}}$ is the channel singular matrix. The received user SINR levels are significantly enhanced with the number of antennas
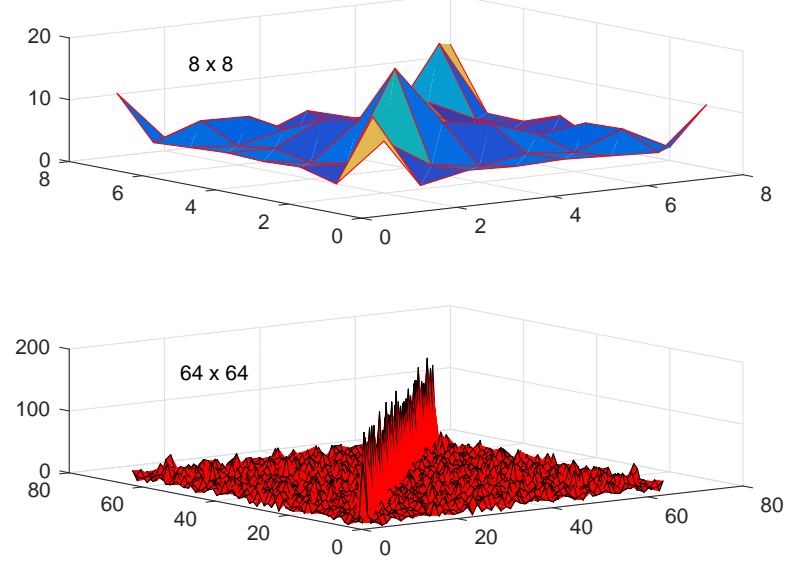

Fig. 6. Channel hardening of $\mathrm{H}^{\mathrm{H}} \mathrm{H}$ with $\left(N_{t}, M_{r}\right)$ setup.

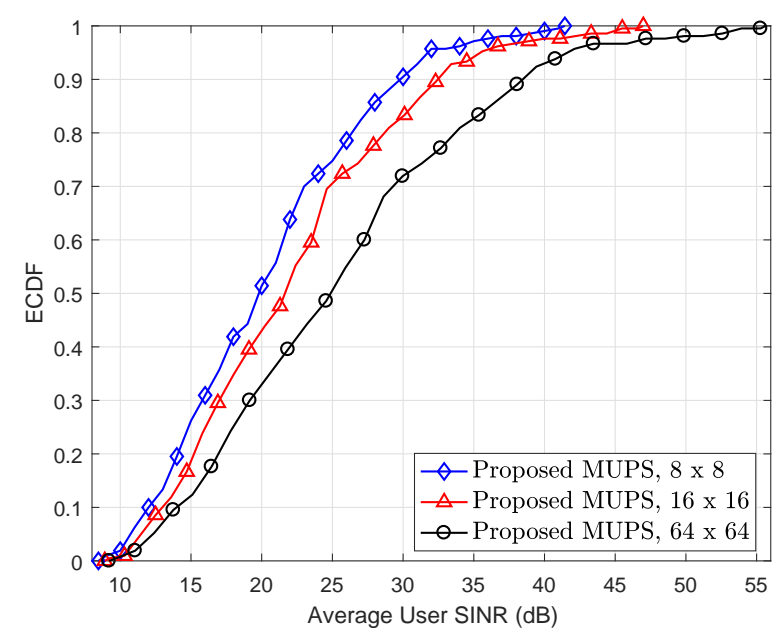

Fig. 7. User received SINR with $\left(N_{t}, M_{r}\right)$ setup.

due to the channel hardening effect. Consequently, further more MU successful pairing events can be achieved with sufficient spatial separation.

\section{Conclusion}

In this work, a joint multi-user preemptive scheduler (MUPS) has been proposed for densely populated 5G networks. Proposed scheduler operates efficiently with different traffic types, e.g., full buffer enhanced mobile broadband (eMBB) and sporadic ultra-reliable low-latency communication (URLLC) traffic. MUPS cross-optimizes the network performance such that the maximum possible spectral efficiency and ultra low latency are simultaneously achievable. Using extensive system level simulations, the proposed scheduler provides significant performance gain, e.g., $62 \%$ gain in average cell throughput, under different network configurations. The performance of the MUPS scheduler is shown to improve with the number of eMBB users until the interference levels become dominant. Hence, proposed conservative MUPS shows further enhanced MU gain by limiting the inter-user interference. Furthermore, 
increasing the number of antennas is shown to harden the wireless channel and thus, further improved URLLC performance can be satisfied. A detailed study on the robustness of the URLLC performance under such a scenario will be considered in a future work.

\section{REFERENCES}

[1] NR and NG-RAN overall description; Stage-2 (Release 15), 3GPP, TS 38.300, V2.0.0, Dec. 2017.

[2] Study on new radio access technology; Radio access architecture and interfaces (Release 14), 3GPP, TR 38.801, V14.0.0, March 2017.

[3] Study on scenarios and requirements for next generation access technologies (Release 14), 3GPP, TR 38.913, V14.3.0, June 2016.

[4] IMT vision - "Framework and overall objectives of the future development of IMT for 2020 and beyond", international telecommunication union (ITU), ITU-R M.2083-0, Feb. 2015.

[5] E. Dahlman et al., "5G wireless access: requirements and realization," IEEE Commun. Mag., vol. 52, no. 12, pp. 42-47, Dec. 2014.

[6] B. Soret, P. Mogensen, K. I. Pedersen and M. C. AguayoTorres, "Fundamental tradeoffs among reliability, latency and throughput in cellular networks," in Proc. IEEE Globecom, Austin, TX, 2014, pp. 1391-1396.

[7] K. I. Pedersen, G. Berardinelli, F. Frederiksen, P. Mogensen and A. Szufarska, "A flexible $5 \mathrm{G}$ frame structure design for FDD cases," IEEE Commun. Mag., vol. 54, no. 3, pp. 53-59, March 2016.

[8] Q. Liao, P. Baracca, D. Lopez-Perez and L. G. Giordano, "Resource scheduling for mixed traffic types with scalable TTI in dynamic TDD systems," in Proc. IEEE Globecom, Washington, DC, 2016, pp. 1-7.

[9] G. Pocovi, B. Soret, M. Lauridsen, K. I. Pedersen and P. Mogensen, "Signal quality outage analysis for URLLC in cellular networks," in Proc. IEEE Globecom, San Diego, CA, 2015, pp. $1-6$.

[10] G. Pocovi, B. Soret, K. I. Pedersen and P. Mogensen, "MAC layer enhancements for ultra-reliable low-latency communications in cellular networks," in Proc. IEEE ICC, Paris, 2017, pp. 1005-1010.

[11] K.I. Pedersen, G. Pocovi, J. Steiner, and S. Khosravirad, "Punctured scheduling for critical low latency data on a shared channel with mobile broadband," in Proc. IEEE VTC, Toronto, 2017, pp. 1-6.

[12] G. C. Buttazzo, M. Bertogna and G. Yao, "Limited preemptive scheduling for real-time systems: a survey," IEEE Trans. Ind. Informat., vol. 9, no. 1, pp. 3-15, Feb. 2013.

[13] Y. Ohwatari, N. Miki, Y. Sagae and Y. Okumura, "Investigation on interference rejection combining receiver for space-frequency block code transmit diversity in LTE-advanced downlink," IEEE Trans. Veh. Technol., vol. 63, no. 1, pp. 191-203, Jan. 2014.

[14] Evolved universal terrestrial radio access (E-UTRA); Physical layer procedures (Release 12), 3GPP, TS 36.213, V12.4.0, Feb. 2015

[15] T. L. Narasimhan and A. Chockalingam, "Channel hardeningexploiting message passing receiver in large-scale MIMO systems," IEEE J. Sel. Topics Signal Process., vol. 8, no. 5, pp. 847-860, Oct. 2014.

[16] A. A. Esswie, M. El-Absi, O. A. Dobre, S. Ikki and T. Kaiser, "A novel FDD massive MIMO system based on downlink spatial channel estimation without CSIT," in Proc. IEEE ICC, Paris, 2017, pp. 1-6.

[17] D. W. Browne, M. W. Browne and M. P. Fitz, "CTH07-4: singular value decomposition of correlated MIMO channels," in Proc. IEEE Globecom, San Francisco, CA, 2006, pp. 1-6. 